\title{
EN DEFENSA DEL MUNDO. NOTAS CRÍTICAS A LA ONTOLOGÍA HIPERPLURALISTA DE MARKUS GABRIEL
}

\author{
IN DEFENCE OF THE WORLD. \\ CRITICAL NOTES ON MARKUS GABRIEL'S HYPERPLURALIST ONTOLOGY \\ Juan Antonio Negrete Alcudia
}

Markus Gabriel, Por qué el mundo no existe, Barcelona: Pasado y Presente, 2015, 228 pp.

$\mathrm{El}$ joven y precoz profesor Markus Gabriel se ha convertido ya en una figura sobresaliente del que es, seguramente, el más visible y seguro de sí de entre los programas filosóficos "continentales" del siglo XXI, el Nuevo Realismo. Lo que sigue son unas notas críticas a algunos aspectos centrales de su concepción ontológica.

La propuesta filosófica de Gabriel es, podríamos decir, un Hiperrealismo hiperpluralista (la primera parte de la denominación la usa él mismo; "jungla ontológica" llama su compañero italiano de realismos, M. Ferraris, al segundo aspecto); o, quizá sería mejor decir, un Hiperpluralismo hiperrealista, ya que, a mi juicio, es más relevante filosóficamente el aspecto pluralista de su tesis que el aspecto realista (y esto vale, tal vez, para todo el debate entre realismo e irrealismo, que esconde dialécticas más importantes: los pensamientos de la diferencia, por ejemplo, son antes un pluralismo o hiperpluralismo que un antirrealismo o "correlacionismo" — como los llama Quentin Meillasoux-). Se podría, pues, decir que, al problema más antiguo (el de lo Uno y lo Múltiple), Gabriel da la respuesta más moderna, o sea, el pluralismo radical, mientras que al problema más moderno (el de Realidad y Representación), Gabriel da, en cambio, la respuesta más antigua, el realismo, aunque en una versión muy cruda, que no fue sostenida quizá por ningún filósofo antiguo, exceptuando quizá a Nietzsche y a Wittgenstein. Estas notas críticas se limitarán al primer asunto.

Aunque los textos de Gabriel se centran en la ontología y la epistemología, sus tesis no dejan de tener importantes implicaciones de todo tipo, dado que, como 
él mismo señala, cualquier otra rama de la filosofía, por ejemplo la Ética, depende de (o, al menos, es especialmente coherente con) una concepción ontológica. No obstante, esas implicaciones no están, a veces, más que insinuadas, y sería deseable que el autor las desarrollase explícitamente en el futuro.

$* * * *$

Empecemos por recordar, con cierto detenimiento, lo que Gabriel nos dice acerca de la Realidad y su pluralidad. Seguiré principalmente, para ello, su libro más importante y exitoso hasta la fecha, y que leo en la traducción francesa, Pourquoi le monde n'existe pas, éditions Jean-Claude Lattès, 2014 (el original alemán es Warum es die Welt nicht gibt, Ullstein, 2013), aunque hay observaciones muy importantes, y no contenidas en ese libro, en otros suyos como Il senso dell'esistenza, Carocci editore, 2013, y en su estudio del idealismo alemán, especialmente del tardío Schelling, Trancendental Ontology, Bloomsbury, 2013.

Gabriel anuncia, desde el comienzo del prólogo-sumario de su libro principal, sus dos tesis ontológicas centrales:

1. El Mundo no existe. Aunque, por fortuna — digamos-, existe todo lo demás, incluso lo que "no existe" (excepción hecha del propio Mundo, por supuesto, que es "menos que nada"): Tesis Hiperpluralista.

2. Conocemos la realidad en sí, y no una mera construcción, y esa realidad incluye a las apariencias de las cosas. Este es el elemento neo-realista, en su versión Hiperrealista.

Hay que mantenerse alejados, pues, según Gabriel, de dos caminos erróneos: la metafísica y la posmodernidad. La metafísica era realista, pero intentaba comprender el Mundo como un todo único, sistemático y ordenado (en este sentido, el naturalismo realista es una concepción perfectamente metafísica). El sujeto humano parece no tener auténtico lugar ahí, pues es reducido a una pieza más dentro del sistema de objetos. Contra esto, valdrá la tesis pluralista: no hay un único mundo omniabarcante. La postmodernidad, por su parte, muy narcisistamente, dice que las cosas solo existen como se nos presentan o las construimos "nosotros". Contra esto hay que defender el realismo: tenemos acceso real a las cosas en sí mismas. Lo que necesitamos, dice Gabriel, es una nueva caracterización o definición de la Existencia.

Para ello, Gabriel parte del que considera el elemento fundamental de la ontología: los Campos de Sentido. Un Campo de Sentido es el lugar donde "aparece" una cosa. 'sentido' tiene aquí, básicamente, el sentido fregeano (bedeutung) de modo de 
darse o ser concebible una cosa, aunque tomado de manera menos determinista y determinable. Las cosas se presentan en diferentes modos: Venus es tanto la estrella matutina como la vespertina; $3+1$ y 2+2 son sentidos de la misma cosa... ¿Cómo llegamos a (la necesidad de) esta noción de Campo de Sentido? Los objetos, señala Gabriel, se distinguen por sus propiedades. Pero ni existe un Superobjeto que contenga todas las propiedades, ni es verdad tampoco que cada objeto se diferencie absolutamente de todos los demás:

No existe un superobjeto (el objeto Todo) porque, de existir, no podría distinguirse de los "otros" objetos, ya que tendría las propiedades de todos. Esto se puede expresar también, mediante el lenguaje de la mereología (estudio de los todos y las partes), diciendo que una cosa no es igual a la suma mereológica de sus partes. Por ejemplo, una estatua o (el cuerpo de) una persona no son igual a la suma de sus partes, pues no pueden recolocarse sin que afecten a la estatua o la persona, ni permiten individualizarla o distinguirla. En todo momento individualizamos o distinguimos objetos (como mi cuerpo, la mesa, el lápiz...), y rechazamos otras posibles individualizaciones (no creemos que mi mano cortada cogiendo un lápiz sean un objeto único). ¿Con qué criterios hacemos cada individualización? Según Gabriel, no hay criterio a priori, no hay un algoritmo: solo la experiencia nos enseña a hacerlo. Hay múltiples catálogos posibles de las cosas. Pero si hubiera un superobjeto, carecería de criterio individualizador posible, pues contendría todas las características.

Tampoco existe una diferencia absoluta entre cada cosa y todas las demás. Una diferencia absoluta de un objeto, es decir, tal que ese objeto se diferenciase de absolutamente todos los otros objetos, reduciría a cero la información acerca de él: solo sería lo que no es ninguno de los otros, pero esto es igual a nada. Las diferencias no son absolutas, sino relativas a un contexto, en el que aparece el objeto. Esta es la verdad del dictum derridiano según el cual no hay nada fuera del texto.

La Existencia es, entonces — he aquí la definición que proporciona Gabriel—, la ocurrencia gracias a la cual cierta cosa se manifiesta en un Campo de Sentido. O, de otra forma, es la aparición en un campo de sentido. (Un Campo de Sentido, por cierto, no es lo mismo que un Dominio de Objetos: los dominios de objetos tienen bien definidos sus objetos, mientras que un Campo de Sentido no define exactamente, por lo que es un concepto más amplio). Pues bien, la Existencia no es una propiedad de las cosas, sino de los Campos de Sentido: la propiedad de que algo surja en ellos. (Ya Frege sostuvo que la existencia es una propiedad de segundo orden, aunque su definición conjuntista es incorrecta, cree Gabriel, pues reduce todo al conjunto vacío). 
Ahora que sabemos lo que es la Existencia, podemos ver más claramente por qué el Mundo no existe. El Mundo es, por definición, el Campo de Sentido de todos los campos de sentido. Pero, por eso, el Mundo no se da en ningún otro campo de sentido, y, por tanto, no existe, pues existir es darse en un campo de sentido. Comprender el Mundo sería comprenderlo como solo una parte de sí mismo, lo que es contradictorio. Este es un resultado análogo al Teorema de Cantor sobre la imposibilidad de un Conjunto de todos los conjuntos. Como en el film Cube de Vincenzo Natali, fuera de todos los cubos, relacionados unos con otros, no hay nada.

"El Mundo no existe" es la primera proposición de la Ontología Negativa. Pero esto implica también proposiciones positivas. La primera de ellas es que "Hay una infinidad de campos de sentido". Además — segunda proposición positiva de la Ontología gabrieliana-, todo campo de sentido es un objeto. Pero esto implica que no hay un único campo de sentido. Como en la serie Seinfeld (literalmente, "campo de ser"), todo es un show about nothing. No hay un superpensamiento, como creía Hegel.

La Pluralidad de campos de sentido nos permite también, según Gabriel, abordar un viejo enigma de la ontología: ¿qué hay de los enunciados negativos? ¿Existen, o no, las brujas? Puesto que hablamos de ellas, parecen existir; sin embargo, lo que decimos de ellas es precisamente que no existen. La historia de la filosofía no ha dejado de dar vueltas a este viejo y enredoso enigma (la "barba de Platón”, según la llamó Quine, sobre la que la navaja de Occam se ha mellado una y otra vez). La solución consiste, nos dice Gabriel, en comprender que la existencia es interna a un campo de sentido, así que la inexistencia es también relativa a un campo: las brujas existen en su campo de sentido, y no existen en otros. No existen trolls en Noruega, pero sí en la mitología nórdica. Incluso los triángulos cuadrados existen, solo que en otro dominio, no en el de la matemática (al menos, tal como es axiomatizada convencionalmente). La inexistencia es no-existencia en un determinado campo de sentido, pero existencia en otro. Esto es lo que ya dijo Platón cuando caracterizó al no-ser como diferencia, como meramente relativo.

Una de las teorías que más insistentemente combate Gabriel a lo largo de todo el libro es el naturalismo reduccionista o cientificismo. El cientificismo es simplemente falso porque, como sabemos, no hay una única Visión del Mundo. Como ha señalado recientemente Putnam, dice Gabriel, tras el naturalismo se esconde el miedo a las hipótesis irracionales. Pero el naturalismo tira al niño con el agua de la bañera. El monismo naturalista es incompatible con el hecho de que las cosas se identifican mediante lo que Saul Kripke llamó "designadores 
rígidos", es decir, significantes que tienen la misma referencia en todo mundo posible. Margaret Thatcher es la misma de la que podemos plantearnos cómo habría reaccionado ante la actual crisis, de modo que su identificación es lógica, no material. Putnam ha argumentado, igualmente, que yo no puedo ser lo mismo que mis partículas, pues en ese caso habría existido antes de nacer, ya que ellas existían en otra configuración.

Si no es la ciencia la que se ocupa del sentido, ¿quién lo hace? Gabriel dedica los capítulos 5 y 6 de su libro a defender que la religión y el arte tienen precisamente aquí su sitio. No me ocuparé de esto.

Que a su definición de Existencia Gabriel le da una importancia mucho mayor que la de un mero ejercicio teórico, en tanto que respuesta a lo que, según nos dice desde el principio, sigue siendo la cuestión de la filosofía, esto es, la cuestión de qué significa todo esto, qué sentido tiene esta vida..., lo muestran claramente algunos párrafos finales:

“El sentido del ser, el significado de la expresión 'ser', o, más bien, 'existencia', es el sentido mismo (...) El hecho de que existe una plétora de sentidos que podemos (re)conocer y transformar, es ya el sentido. O, para ir a lo esencial: el sentido de la vida, es la vida, la confrontación con el sentido infinito, en la que por fortuna tenemos el derecho de participar. Al hacerlo, que no seamos siempre felices se comprende fácilmente. Que existe desgracia y dolor inútil es hasta tal punto verdadero, que debería ser la ocasión de pensar de nuevo el ser-hombre y de mejorarnos moralmente (...) El paso siguiente consiste en olvidar esa búsqueda de una estructura fundamental englobante para intentar, en su lugar, de manera colectiva, comprender mejor las numerosas estructuras existentes, con menos toma de partido previa, de manera más creativa, a fin de ser aptos de juzgar mejor lo que puede quedar y lo que hay que cambiar, pues no es que porque todo exista, todo esté bien. Nos encontramos todos juntos en una gigantesca expedición, llegados aquí de ninguna parte, avanzamos juntos en el infinito". (Pourquoi le monde n'existe pas, pg. 279 — traducción mía—)

$* * * *$

Pasemos ya a nuestras observaciones críticas a la tesis hiperpluralista de Markus Gabriel.

Según Gabriel, la noción fundamental de la Ontología, "Campo de Sentido", es la noción de algo radicalmente plural, es decir, es una noción que se aplica a una pluralidad irreducible de objetos, sobre los cuales no hay unidad alguna. Dicho en viejos términos, el Ser o la Realidad no es uno en ningún sentido o grado (al 
menos en ninguno ontológicamente relevante). Ahora bien, el pluralismo radical está afectado por importantes aporías.

Empecemos por esta pregunta: ¿qué significa la expresión misma "Campo de Sentido"? Preguntar esto es, o debería ser, lo mismo que preguntar qué tienen en común todos los campos de sentido. Es lo que preguntamos cuando preguntamos qué significa 'ser' o 'realidad' aplicado a los diferentes ámbitos de ser o de realidad... Es evidente que en la ontología de Gabriel "Campo de sentido" es un concepto de aplicación sumamente universal. Pero ¿`se usa unívocamente, equívocamente, o analógicamente?

Si tiene un sentido unívoco, entonces hay algo, y algo esencial (la esencia misma de todo campo de sentido) que todos los campos de sentido tienen en común. Campo de Sentido sería, en ese caso, el género de toda realidad, del cual cada campo de sentido o aspecto de la realidad, sería una especie. De hecho, Gabriel da una única definición de 'Campo de Sentido' ("lugar en que aparece cualquier cosa" Pourquoi le Monde n'existe pas, pg. 286), que debe servir para todos ellos. Podría decirse, a lo sumo, que hay una diferencia "material" o de contenido entre unos campos y otros, pero la forma sería idéntica en todos ellos. Y sería, entonces, preciso embarcarse en la discusión de la relación entre la forma y la materia, en la relevancia ontológica de la forma, etc. En cualquier caso, no se podría decir simplemente que la realidad es múltiple y que no es una, esto es, no podría decirse simplemente que el Mundo no existe.

Aquí hay la gran tentación de responder que el concepto de Campo de Sentido es, eso, "solo un concepto", es decir, una manera en que nosotros comprendemos la realidad, no algo que tenga que corresponderse diáfanamente con algo real. Sin embargo, esta tentación (que se llama Conceptualismo) es seguramente la tentación en la que menos querría caer un realista extremo, es decir, alguien que cree que nuestro conocimiento de la realidad no está mediado por nuestras maneras de entenderla. ¿Precisamente la noción fundamental de la ontología sería algo que no se corresponde con ninguna realidad o aspecto relevante de ella, pues no existe un campo de sentido genérico?

Hay razones para rechazar el univocismo en ontología. Aristóteles (entre otros) vio que 'ser' no puede ser unívoco, es decir, que la Realidad no tiene unidad de forma simple y completamente unívoca, pues esto la reduciría a solamente una (el ser-uno de Parménides), y Aristóteles (como ya Platón) encuentra insatisfactorio e inconsistente este monismo extremo: no solo no salva los hechos (la naturaleza), sino que cae en contradicción, pues quien piensa y habla, implica ya la diferencia. Si 'ser' fuese unívoco, las cosas solo podrán distinguirse por el no-ser... (Eso, 
suponiendo que el propio no-ser escapara al género ser; pero Gabriel, según hemos visto, no admite que el no-ser sea exterior al ser; $y$, además, no cree que las diferencias puedan darse en el campo total, sino solo en campos parciales: ninguna cosa se opone a todas las demás).

¿Entonces, el concepto de Campo de Sentido, con el que nos referimos a las múltiples realidades irreducibles que, según Gabriel, pueblan la infinitud anaximandrea, se dice equívocamente? Esta parece la opción más coherente con el pluralismo ontológico: si las múltiples realidades no pueden ser reducidas, en ningún sentido importante, a una única realidad, entonces tampoco el concepto de realidad o Campo de Sentido puede reducirse a uno: si los sentidos son múltiples, 'Sentido' no puede tener un único sentido ni sentidos reducibles. Gabriel estaría llevando a su extremo el equivocismo moderno, que, en el ámbito de la filosofía analítica, se canonizó como la distinción de diferentes "valores de ser": el cuantificador existencial, la predicación, la identidad...

Sin embargo, el equivocismo es todavía más destructivo que el univocismo. Bajo el supuesto equivocista, cada vez que Gabriel usa la expresión 'campo de sentido' lo estaría usando de manera similar a como usamos el término 'gato', ora referido al felino ora a la máquina. $\mathrm{Y}$ recordemos que hemos rechazado la escapatoria conceptualista. El equivocismo es una manera aparentemente afirmativa de realmente negar el campo de objetos al que se refiere. Si nada hay en común entre los diversos usos de la misma palabra, realmente no es la misma palabra, o, si se quiere, es solo eso: una misma palabra, sin contenido ni sentido.

Parece que solo queda encaminarse a alguna versión analogista de la Ontología. Aún dentro de esta opción, quizá quepa distinguir entre un analogismo-pluralista o inclinado del lado de la pluralidad, y un analogismo-monista o inclinado a la unidad. Sería un ejemplo de este segundo el analogismo de Aristóteles (referido a las categorías) y el platónico-tomista (referido también a la jerarquía de las sustancias). Un ejemplo del primero sería el analogismo (si lo es) de la tesis de los "aires de familia" del segundo Wittgenstein. Es claro que sería un analogismo de este tipo el que más le convendría a Gabriel, pues el otro es solo un monismo moderado, o una síntesis de monismo y pluralismo. Según este tipo "wittgensteiniano" de respuestas a la cuestión de la unidad o pluralidad de sentidos de 'ser' o, en nuestro caso, de 'campos de sentido', unos "juegos de lenguaje" y unas formas de vida, se parecen a otros, y estos, a su vez, a otros, pero, a lo largo del desplazamiento, unos ya no tienen por qué conservar ningún parecido con aquellos con que empezábamos. Como en los juegos: ¿qué tienen en común todos los juegos?, se preguntaba Wittgenstein. Nada, más que a lo sumo vacuidades (como "tener 
reglas"). Algo así podría decirse de los Campos de Sentido: todos tienen reglas, sí, pero incluso eso se diría de manera feblemente analógica.

Las posiciones analogistas me parecen, aunque más contraintuitivas para el sentido común, también menos evidentemente aporéticas que las posiciones extremas (univocismo y equivocismo). De hecho, en alguna de ellas es donde se está más cerca de la verdad, a mi parecer. Ahora bien, incluso si se elije una versión de lo que he llamado analogismo-pluralista, ya no se puede decir simplemente que "el Mundo no existe". A lo sumo, se podrá decir que el Mundo, o Campo de Sentido de todos los campos de sentido (o, en lenguaje wittgensteiniano, el Juego de Lenguaje de todos los juegos de lenguaje) existe menos que los diversos campos de sentido o juegos de lenguaje en que se "divide". En el análisis trascendental en que se consigna la división de juegos de lenguaje, se supone una unidad que es lo que significa Juego y Lenguaje. Salvo que caigamos en el puro equivocismo, y ya no tengamos de qué hablar. Ciertamente, Gabriel (como la mayoría de los filósofos modernos) no se plantea la cuestión de si univocismo, equivocismo o analogismo. Pero este es un vacío en su teoría, pues, aunque nos dice que la Realidad no es analizable matemáticamente, no nos explica qué tipo de intelección es la que le corresponde.

¿Queda a salvo la ontología con un analogismo débil o pluralista? ¿Es posible, en realidad, un analogismo así? Dejaremos esta profunda cuestión para otro momento.

La otra veta desde la que abordar el asunto del pluralismo es el de la relación entre los distintos Campos de Sentido. El antirreduccionismo de Gabriel nos pide que reparemos en que no tiene sentido pretender reducir una charla en un restaurante, a un baile de partículas; o, en otro de sus ejemplos, el mundo de sentido de un niño al mundo de sentido de un adulto. Son heterogéneos, pero todos reales en la misma medida. No debemos empobrecer la realidad, y tampoco podemos, porque el reduccionismo falla, se autocontradice: una teoría científica, por ejemplo la propia teoría de partículas, solo tiene sentido en un Campo de Sentido de pensamientos y debates humanos, no en el campo de las propias partículas que son su objeto.

Esta es una demanda convincente, a mi juicio (aun el viejo reduccionismo naturalista ha dejado paso a versiones más matizadas y complejas). Sin embargo, en su unilateralidad y extremismo, deja de serlo. Porque es evidente que hay relaciones entre Campos de Sentido diversos, y no solo relaciones de solapamiento (que, 
en realidad, es una relación de completa indiferencia), sino relaciones de alguna u otra forma "causales", y relaciones de jerarquía ontológica. Una modificación en el ámbito de las partículas, por ejemplo, produce o, al menos, está correlacionada necesariamente con una modificación en el campo de sentido de nuestra charla en el restaurante (una explosión cercana, acaba con la cena), y también en sentido inverso (mi intención de marcharme acaba con una organización "ceniforme" como diría van Inwagen- de las partículas involucradas en la cena).

Por otra parte, no parece muy consolador, ante nuestra inminente muerte en el Campo de Sentido que consideramos habitualmente más "real" (o más abarcador de nuestros diversos campos de sentido), que se nos diga que no debemos preocuparnos, porque solo ocurrirá que dejaremos de existir o aparecer en ese ámbito, pero existiremos en otros (en el de quienes nos imaginen, por ejemplo). Así es como "existen" los trolls, sí...

Hay, pues, relaciones de causalidad (de superveniencia, etc.) y grados o modos de existencia más relevantes que otros. Relativizar la Existencia diciendo que ningún Campo de Sentido es "más real" que otro, no es convincente. Si aceptamos que algunos Campos de Sentido determinan a otros, entonces nos vemos conducidos otra vez al problema de si tiene que existir, y cómo, el Campo de Sentido que determina a los demás y no es determinado por otros.

Gabriel, como dijimos, ofrece dos argumentos por los cuales se probaría que no puede haber un único Campo de Sentido:

El primero dice que no puede existir un Campo de Sentido que abarque a todos los campos de sentido, pues una cosa (sea un simple objeto o un campo de sentido) solo existe si aparece en un entorno de otros objetos y hechos, y no hay (por principio) un campo superior en que aparecería el Mundo. No puede existir, en la imagen de la película Cube, un Cubo de todos los cubos (esto nos confirma, por cierto, que Gabriel no quiere caer en la tentación conceptualista, pues si fuese lícita la posibilidad de comprender algo desde una noción que, siendo más abarcadora, no tuviese un equivalente importe ontológico, no habría problema para entender al Mundo dentro de un campo de sentido que fuese mayor epistemológicamente pero no ontológicamente).

Creo que ese primer argumento de Gabriel es rechazable si entendemos la Existencia y el Campo de Sentido de manera no extensional sino intensional, como de hecho creo que tenemos que entender todas las nociones ontológicas. Para que se entienda qué quiero decir, pensemos en el argumento en el que se inspiran todo este tipo de radicales pluralismos, contingentismos, etc., como el de Badiou, Meillassoux y el propio Gabriel: me refiero al Teorema de Cantor. Según este teorema, no 
existe un conjunto $\mathrm{U}$ (Universal), que contenga a todos los conjuntos, puesto que el conjunto-potencia, $\mathrm{P}$, de cualquier conjunto $\mathrm{A}$, es mayor que A (el conjuntopotencia de A es el conjunto formado por todos los subconjuntos de A: así, si A es $\{1,2,3\}, P(A)$ es $\{(1),(2),(3),(12),(13),(23),(123)\})$. De aquí se sigue, se dice, que no existe el Conjunto de todos los conjuntos. No obstante, este resultado tiene que ser matizado. Lo que estrictamente se sigue de él, es, a lo sumo, que no existe el Conjunto de todos los conjuntos si definimos a los conjuntos por su extensión. Para explicar esto, pensemos en lo siguiente: efectivamente, no existe un número natural mayor que todos los demás números naturales, pero eso no implica que no exista la Clase o Conjunto de (todos) los números naturales, o sea, N. Esto lo que significa es que $\mathrm{N}$ no se define por extensión, es decir, por la simple enumeración de sus partes. Pero es que una definición por extensión es un absurdo lógico, pues ¿cómo se puede identificar qué miembros deben ser incluidos o enumerados en la extensión? Solo un conjunto completamente arbitrario podría definirse así, un conjunto que se estaría constituyendo en el preciso arbitrario acto de enumerarlo. Lo que define a los conjuntos, clases o géneros en general, es la cualidad o propiedad que todos sus miembros deben compartir. (Es cierto que el problema de la extensionalidad tiene una relación especial con el ámbito de los conjuntos, números, y demás objetos matemáticos, pues es de creer que precisamente la Matemática se define como el estudio de la Extensión. No obstante, podemos dejar ahora esta cuestión, pues Gabriel, a diferencia de Badiou y Meillassoux, no acepta que la Matemática sea un modelo adecuado de la Ontología). Por tanto, en conclusión, está lejos de seguirse que el concepto de ser tiene que o ser equívoco o no existir puesto que iría contra la conclusión cantoriana. El concepto Ser o Realidad no es extensional, sino intensional. También es analógico (y, por eso, dialéctico). Aplicado todo esto a los Campos de Sentido, ¿en qué sentido puede decirse, en ontología, que el Campo de Sentido de todos los campos de sentido "engloba" a todos los "demás"? El Campo de Sentido de todos los campos de sentido es aquel que da sentido al hecho de que haya campos de sentido, es decir, a la propia existencia de campos de sentido.

Por supuesto, el concepto de Ser o Realidad, o de Campo de Sentido, es absolutamente singular, o, mejor dicho, la Singularidad en sí. En este sentido, se puede decir que no es comprensible. La Existencia misma (el ipsum esse), ha dicho siempre la mejor metafísica tradicional, es, en cierto modo, inconceptualizable (epekeina tes ousías), precisamente porque es la concebibilidad misma.

Con este resultado, podemos dirigirnos al otro argumento de Gabriel contra la existencia del Mundo: cualquier concepción del Mundo — dice este otro argumento- sería interna a él, luego no podría abarcarlo (sería como intentar ver 
el bosque desde dentro). No puede contemplarse al Todo desde dentro, y aquello que no podemos contemplar apareciendo, no existe (por cierto, esto, y como ha señalado F. Nef, le da un sesgo muy epistemológico al "realismo" de nuestro autor).

Como se sabe, los filósofos discuten desde siempre si se puede comprender y/o entender lo Infinito. Aunque el Infinito tradicional era más domesticable que el Infinito cantoriano, el viejo infinito era ya lo suficientemente "grande" (y pequeño, como señaló Cusa, por ejemplo) como para que ningún ser finito lo abarcase. Ahora bien, suponiendo que este problema fuera realmente insalvable, no afectaría menos a la teoría ontológica de Gabriel que a la de, por ejemplo, Descartes, puesto que, según Gabriel, tenemos que aceptar que existe realmente una infinidad indomesticable de Campos de Sentido. Si tenemos que aceptar eso es porque, de alguna manera, podemos comprenderlo. Desde luego, se puede decir que este es un comprender completamente negativo. Esto es lo que siempre dijo la teología negativa. Pero, si desde dentro de una pluralidad infinita de campos de sentido, podemos entender esa pluralidad, entonces desde dentro de una Unidad del Campo de Sentido podemos entender, al menos negativamente, esa unidad. Dios es un asunto místico, también para los racionalistas más conscientes.

\author{
Juan Antonio Negrete Alcudia \\ http:/ / wnw.dialecticayanalogia.blogspot.com \\ dialecticayanalogia@gmail.com
}

